\title{
De alianzas, contextos y biografías. Una experiencia académica "internacional" con Brasil desde el noroeste Argentino
}

De alianças, contextos e biografias. Uma experiência acadêmica "internacional' com Brasil desde o noroeste Argentino

About alliances, contexts and biographies. An "international" academic experience with Brazil from northeastern Argentina

\section{Mariana Inés Godoy}

Doctora en Antropología (UBA-Arg.) y Socióloga (UNSE-Arg). Investigadora Asistente del CONICET con sede en el Instituto de Investigaciones en Ciencias Sociales y Humanidades (ICSOH-CONICETUniversidad Nacional de Salta).

\section{Resumen}

En este ensayo procuro hacer un ejercicio de reflexividad sobre un recorrido de formación académica y en investigación en el campo de la antropología jurídica y la administración de conflictos, que estuvo especialmente marcado por experiencias de vinculación con parte de la academia brasileña. Viniendo de provincias del noroeste argentino, lejos de los grandes centros académicos y urbanos, estas experiencias de vínculo internacional muestran un contexto de una relativa expansión de los sistemas de becas doctorales en Argentina y del financiamiento en ciencia y técnica en general. Al mismo tiempo, permiten pensar en cierta dinámica en los equipos de investigación que también animan movimientos disciplinares. Estas alianzas internacionales, que tienen componentes de inversión académica aunque también de índole afectiva, se alimentan y se consolidan en esos escenarios de crecimiento. De igual manera, actúan compensando movimientos inversos de retracción de la inversión en el sistema científico, permitiendo sostener e incentivar la producción, y adquiriendo el sentido de resistencias políticas a esos escenarios adversos.

Palabras claves: Campo científico, Alianzas, Administración de conflictos, Argentina, Brasil. 


\section{Resumo}

Neste ensaio procuro fazer um exercício de reflexividade sobre uma trajetória de formação acadêmica e de pesquisa no campo da Antropologia Jurídica e da administração de conflitos, que esteve especialmente marcado por experiências de vinculação com parte da academia brasileira. Vindo de estados do noroeste argentino, longe dos grandes centros acadêmicos e urbanos, essas experiências de vínculo internacional evidenciam um contexto de relativa expansão dos sistemas de bolsas de doutorado na Argentina e do financiamento em ciência e técnica em geral. Ao mesmo tempo, permitem pensar em certa dinâmica dos grupos de pesquisa que também aninam movimentos disciplinares. Essas alianças internacionais, que têm componentes de inversão acadêmica, mas também de tipo afetivo, são alimentados e consolidados nesses cenários de crescimento. Da mesma forma, compensando movimentos contrários de retração da inversão no sistema científico, permitem apoiar e incentivar a produção, adquirindo o sentido de resistências políticas diante desses cenários adversos.

Palavras-chave: Campo científico, Alianças, Administração de conflitos, Argentina, Brasil.

\section{Abstract}

This essay aims to make a reflexion exercise on a period of academic training and research on the field of law anthropology and conflict management that was especially marked by correlation experiences with some Brazilian academics. Considering that these experiences come from provinces in the northwest of Argentina far from the big academic urban centers, it shows the occurrence of a certain expansion in the allocation system of doctorate scholarships in Argentina, together with a better financing of Science and Technology in general. At the same time, these international alliances imply a degree of dynamism in the research teams that encourage disciplinary movements. They have components both of academic investment and of affective relations, which nurture and consolidate in development scenarios. Furthermore, they compensate retraction movements on the investment in the scientific field, thus allowing the motivation and maintenance of academic productions, which act as political resistance to adverse policies.

Keywords: Scientific field, Alliances, Conflict management, Argentina, Brazil. 


\section{INTRODUCCIÓN}

En este relato intento hacer un ejercicio de reflexividad sobre un recorrido de formación académica y en investigación en el campo de la antropología jurídica y la administración de conflictos, que estuvo especialmente marcado por experiencias de vinculación con parte de la academia brasilera. Ese recorrido, iniciado en 2011, a partir de una estancia como estudiante de doctorado en cuya tesis trabajé sobre la prolongación de un conflicto político en la arena judicial, se extendió hasta la actualidad, con la conformación de una alianza de trabajo en torno al campo de la mediación y los métodos alternativos de administración de conflictos. En ambos trabajos mis contextos de reflexión fueron provincias del noroeste argentino, Santiago del Estero y Salta, distantes de los grandes centros urbanos. En el NOA y el NEA, están las provincias que vulgarmente se aluden como el "interior" las más "atrasadas", coloniales, rurales y a las que siempre se nombran con los índices más problemáticos en cuanto a pobreza, tasas de analfabetismo, desnutrición, violencia de género... ${ }^{1}$ Allí también se situaron mis lugares institucionales de trabajo, también retirados de los grandes polos académicos, pero que intentaban afirmarse y adquirir su propia identidad mientras se nutrían de nuevos becarios e investigadores.

Cabe advertir que este relato no necesariamente refleja el modo en que fueron vividas y significadas estas experiencias por parte de los distintos involucrados en estas redes de formación y trabajo. La exposición de parte de esta trayectoria personal, cual un "caso", enseña de algún modo -todavía nos merecemos un estudio riguroso, estadístico pero también cualitativo al respecto- el impacto de una política de expansión de los programas de posgrado y de los sistemas de beca en Argentina -y Brasil- en las trayectorias académicas y sociales de cientos de jóvenes. Y alertan sobre ciertos procesos de apertura de nuestro campo científico a partir de la "irrupción de nuevos ocupantes provistos de nuevos recursos", (BOURDIEU, 2003, p. 69) de re-equilibrios de

\footnotetext{
La región Noroeste, según datos del Censo de 2010, concentra un 11,4\% de la población del país, el 15,9\% de Hogares con NBI (frente al 8,3\% de la Región Metropolitana) y una tasa de analfabetismo del 8,6\% (frente al 5,6\% de la Región Metropolitana). Extraído de FACHELLI; LÓPEZ-ROLDÁN, 2017.
} 
poder entre las disciplinas y grupos de investigación, aunque también donde se consolidan y hacen más fuertes algunos de los grupos existentes. Es cierto que, como también dice Bourdieu, existe una relación eficaz entre las propiedades de origen social de los agentes científicos y sus estrategias y posición adquirida en ese campo (de esta jerarquía previa dependen además, según el autor, todas las orientaciones en cuanto a disciplina, una especialidad e incluso "estilo" científico del agente (BOURDIEU, 2003, p. 80). Sin embargo, nuestra hipótesis es que esa relación puede claramente afectarse por una política global de redistribución de recursos. Esto también nos da a pensar sobre cuánto podemos sostener la premisa de Bourdieu de la autonomía del campo científico o cuánto un exceso de autonomización implica necesariamente su contracción y cierre y, en definitiva, su pérdida de dinamismo².

Por otro lado, también sostenemos la premisa, siguiendo en parte la propuesta del sociólogo Michel Callon (1995), de que la producción de conocimiento y aún la configuración de problemas de investigación, es simultánea a la construcción de redes de relaciones, alianzas y negociaciones entre colegas científicos y a la vez con nuestros propios interlocutores en el campo-aunque él se referiría a elementos de la naturaleza como objetos de estudio-. Es decir que, si bien existen disponibles antecedentes académicos en ciertas materias de pesquisa, esos antecedentes se vuelven materia viva y de algún modo hacen emerger nuevos problemas cuando por la naturaleza de nuestra propia tarea como investigadores producimos distintas interacciones sociales.

Esas alianzas de trabajo configuran entonces nuestras posibilidades de pensar objetos y problemas de indagación y también ayudan a contrarrestar escenarios adversos que minan la producción individual, hasta transformarse en verdaderos lugares de resistencia política.

\footnotetext{
El autor explica que el campo científico obedece a una lógica que no es la del campo político (p. 99). Y que si bien está sometido a presiones exteriores, es relativamente autónomo respecto al universo social que lo rodea -hubo un proceso histórico largo de autonomización-, por lo tanto "dispone de la libertad necesaria para desarrollar su propia necesidad, su propia lógica, su propio nomos” (p. 87) regulando completamente el derecho de admisión impuesto a los aspirantes a ingresar en él (de hecho la autonomización implica hacer más difícil aún ese ingreso). Esta autonomía es en parte actuada y reforzada con sus asociaciones, sus reuniones, sus revistas, sus medallas... (p. 92) y estas a su vez permiten incorporar una libido scientifica, la illusio, la creencia en el juego, en lo que está en juego en que la cosa vale la pena, compensa jugarla (p. 93).
} 


\section{LA PRIMERA EXPERIENCIA CON LA ACADEMIA BRASILERA: UNA ETAPA FORMATIVA}

Durante el segundo semestre del año 2011 cursando el último año como becaria doctoral del Consejo Nacional de Investigaciones Científicas y Técnica en Argentina (en adelante CONICET) con sede en el Instituto de Estudios para el Desarrollo Social (INDES), dependiente de la Universidad Nacional de Santiago del Estero, tuve la posibilidad de acceder a una beca de misión de doctorado vinculada al Núcleo Fluminense de Estudos e Pesquisas de la Universidade Federal Fluminense (Niterói-Rio de Janeiro), a partir de mi vínculo como investigadora del Equipo de Antropología Política y Jurídica de la Universidad de Buenos Aires, como tesista dirigida por María Victoria Pita ${ }^{3}$. Acababa de mudarme desde el Doctorado en Ciencias Sociales al de Filosofía y Letras, ambos de la UBA. Este último contenía la orientación en Antropología Social. Después de un recorrido de cuatro años de cursado de seminarios, de largas conversaciones con profesores, compañerxs y mi directora me había convencido que escribiría una tesis que debían leer y evaluar antropólogos.

En esa ocasión participamos siete estudiantes de programas de Maestría y Doctorado en Antropología Social de la Universidad de Buenos Aires y de la Universidad Nacional de Misiones, provenientes de diversas ciudades como Buenos Aires, Rosario (Santa Fe), Posadas (Misiones), Tilcara (Jujuy) y en mi caso, como anticipé, de Santiago del Estero, en la región noroeste de Argentina.

A diferencia de la experiencia de otrxs investigadores, en mi caso no había tenido previamente la iniciativa de postular intercambios internacionales; por alguna razón biográfica salir de Argentina no estaba entre mis esquemas de posibilidades. Tampoco lo habían hecho previamente otros becarios de mi lugar de trabajo. Así es que a mi esta experiencia se me dio como un "regalo" que abriría puertas insospechadas. Llegar a Rio de Janeiro además, fue llegar a una ciudad

\footnotetext{
3 La beca que me llevó a Brasil en esa primera ocasión, se enmarcaba en el ámbito del Convenio Capes/ MINCyT 184/10, entre el Ministerio de Ciencia, Tecnología e Innovación Productiva de Argentina y la Coordenação de Aperfeiçoamento de Pessoal de Nivel Superior de Brasil, bajo el proyecto "Procesos institucionales de administración de conflictos en perspectiva comparada. Brasil-Argentina” coordinado por Sofía Tiscornia, directora del Equipo de Antropología Política y Jurídica de la UBA y Roberto Kant de Lima, en esa época coordinador del NUFEP/UFF.
} 
enorme e intimidante para mí, por eso preferí vivir en Niterói que se asemejaba en su proporción a las ciudades por las que estaba acostumbrada a circular. Esta inseguridad disminuyó a partir de la compañía de mis compañerxs argentinxs y también de la amable recepción de lxs profesores del programa.

Ese semestre lxs siete argentinxs "invadimos" el Programa de Pós-Graduação em Antropologia y el Núcleo Fluminense de Estudos e Pesquisas de la Universidad Federal Fluminense, cuyos docentes e investigadores debieron canalizar la inquietud formativa en torno a problemáticas locales tan diversas como las disputas y conflictos de tierra y recursos de comunidades indígenas en territorios como la Quebrada de Humahuaca, en Jujuy, o de campesinos en el nordeste de Misiones, en la región mesopotámica; las formas de violencia interpersonal de jóvenes de uno de los centros urbanos más grandes del país como Rosario-Santa Fe; los sistemas de acceso a los servicios de salud pública de personas pertenecientes a la comunidad Quom en la misma ciudad de Rosario; prácticas corporales en torno a experiencias de danzas populares en Buenos Aires y ciudades del sur de Brasil...

Convergían en esa experiencia particularmente cuantiosa en la cantidad de argentinos en Brasil la expansión de las Becas CONICET de formación doctoral en Argentina a través de las cuales muchos estudiantes de las provincias del "interior" habían podido acceder a ese nivel de formación; la multiplicación de ofertas de maestría y doctorado en las universidades argentinas, entre ellas la apertura de la Maestría en Antropología Social de la UBA en la localidad de Tilcara ${ }^{4}$; y la capacidad de iniciativa de dos grupos de investigación para gestionar oportunidades de intercambio, diálogo y formación en el área de seguridad pública y administración de conflictos en un escenario de

\footnotetext{
${ }^{4}$ El pueblo de Tilcara, está situado a 2500 m.s.n.m. en la Quebrada de Humahuaca provincia de Jujuy y cuenta con 6 mil habitantes. Allí la Universidad de Buenos Aires tiene un centro universitario que consta de un sitio arqueológico abierto al turismo, un museo arqueológico y antropológico, un instituto interdisciplinario con distintas ofertas de posgrado, proyectos de investigación y una residencia universitaria. La primera cohorte de estudiantes de la Maestría en Antropología Social se abrió en el año 2009 y luego siguieron dos cohortes más. Para quienes residíamos en ciudades del norte argentino esta maestría ofreció un espacio propicio, por su proximidad geográfica y su calidad académica, para tomar distintos cursos de posgrado en antropología a quienes estábamos cursando algún doctorado. Allí conocí en 2009 a María Victoria Pita, a cargo del seminario "Violencias, burocracias y demandas de justicia" y quien se transformó en mi directora de tesis.
} 
ampliación de las estructuras de financiamiento para la internacionalización de la pesquisa y la formación de posgrado. En este último sentido, no se debe desconocer cierta vocación federal del EAPJ por extender estos beneficios fuera de Buenos Aires y del núcleo de investigadores propios.

Para detenernos en el primer punto, la cantidad de becarios doctorales de CONICET con dedicación exclusiva que en el año 2001 habían sido de 300, pasan a 1.095 en 2003, a 4.994 en 2007 y a 7.087 en 2011 (luego a 7.031 en 2013 y 8.413 en 2017) ${ }^{5}$. El Plan Estratégico Nacional de Ciencia, Tecnología e Innovación “Bicentenario" (2006-2010) presentado bajo la presidencia de Néstor Kirchner, proponía como meta para el año 2010 alcanzar una inversión nacional en ciencia y tecnología equivalente al $1 \%$ del Producto Bruto Interno (PBI), teniendo en cuenta que en 2004 esta inversión había sido de un 0,49\%. En cuanto al aumento del número de becas doctorales el diagnóstico indicaba que en Argentina se graduaba un número muy bajo de doctores por año (menos de 500), comparado con países como Brasil y España (donde sobrepasaban los 6.500), cuestión que impedía la posterior orientación al desarrollo de la carrera de investigación. Así, este organismo concentró especialmente desde el año 2005 el concurso y asignación de becas doctorales a jóvenes de hasta 30 años de edad en contraste con el sistema brasilero donde la distribución de becas de estudio está más atada a las estructuras de posgrado de cada universidad. Las convocatorias anuales hasta al menos el año 2016 buscaban un desarrollo armónico entre las distintas disciplinas de las llamadas cuatro grandes áreas de conocimiento: 1) Ciencias Agrarias, de la Ingeniería y de Materiales; 2) Ciencias Biológicas y de la Salud; 3) Ciencias Exactas y Naturales y 4) Ciencias Sociales y Humanidades. Y por otro lado el Plan Estratégico mencionado abogaba por un reequilibrio regional de los recursos, pues, según ese diagnóstico, las cinco circunscripciones territoriales del país más desarrolladas concentraban el $80 \%$ de los recursos de inversión y los recursos humanos del sector científico y tecnológico, mientras que las diecinueve restantes ape-

\footnotetext{
Para esbozar estos datos recurrimos a las publicaciones en la página de CONICET de la Gerencia de Recursos Humanos publicadas en distintos años. Si bien por la fecha de los hechos que relatamos nos interesan especialmente los datos disponibles que describen hasta 2011, en el momento de escribir este artículo sólo se encuentra la comparativa de datos de distinto tipo entre 2007 y 2017 (CONICET, 2018).
} 
nas recibían el $20 \%{ }^{6}$. Ese objetivo efectivamente se fue concretando, y fue en ese espacio donde se facilitaron las condiciones para el ingreso a la formación de doctorado de graduados recientes de provincias del "interior"7.

En mi caso particular me encontraba construyendo un objeto de investigación para mi tesis de doctorado que parecía por momentos el "más exótico" de los presentados en Brasil por mis compañerxs, no encuadrándose del todo en ninguno de los campos de la disciplina en que distintos grupos de investigación habían ido delimitando sus interrogantes y líneas analíticas. El núcleo de investigación brasilero había hecho sus principales aportes en torno a prácticas institucionales de administración de conflictos y al estudio comparado de sistemas judiciales, generalmente, aunque no exclusivamente, a partir de la experiencia de grandes metrópolis (http://www.ineac.uff.br); el EAPJ-UBA había construido su programa de pesquisa en referencia a experiencias de violencia institucional y violaciones de derechos humanos, también con epicentro especial en Buenos Aires (http://www.antropojuridica.com.ar). Mi trabajo se situaba en el cruce entre la antropología de la política y la antropología jurídica, donde concurrían problemáticas como la caída de un líder carismático en un régimen político provincial calificado como "patrimonialista"; el intercambio de "regalos" entre mujeres de una rama de la organización partidaria; un proceso de judicialización de un conflicto político entre esas mismas mujeres en un sector de proximidad a la referente máxima del partido; la superposición de la burocracia administrativa y judicial con el trabajo de la militancia territorial... ${ }^{8}$ Desde mi aproximación a la comprensión antropológica,

\footnotetext{
El objetivo era elevar paulatinamente la participación de las diecinueve circunscripciones más postergadas hasta un 30\% para el año 2010, y a un 40\% para 2015, duplicando de esta forma su participación de 2006.

Como ejemplo de esa evolución en el año 2007 Santiago del Estero contaba con el 0,2\% de becas financiadas por CONICET (10 becas, de un total de 5.599) y Salta con el 0,9\% (48 becas) mientras que la ciudad de Buenos Aires (o Capital Federal) concentraba el 30,8\% de las becas (1726 becas). En 2013 la ciudad de Buenos Aires disponía del 27,70\% de las becas (2462 becas). Hacia 2017, Santiago del Estero ya representaba el 0,9\% de las becas (103 becas de un total de 11.385), Salta llegaba al 2,2\% (251 becas) y ciudad de Buenos Aires el 24\% (2736 becas).

8 Esta tesis doctoral, bajo la dirección de María Victoria Pita, fue defendida en la Facultad de Filosofía y Letras de la UBA en marzo de 2013 y publicado a fines de 2014 como Los regalos de Margarita. Conflictos, trama política y justicia en el juarismo santiagueño, editado por EDUNSE y prologado por Fernando Balbi.
} 
buscaba sacar ese proceso de los prejuicios que sentía habían instalado motes como el de caudillismo, patrimonialismo y autoritarismo.

Habría que documentar el modo en que en esos años se produjo un particular movimiento de interés por la antropología entre estudiantes y graduados de disciplinas como Sociología o Comunicación Social, de modo que mi trayectoria en este marco no ha de ser del todo excepcional. Recordemos que en Argentina, a diferencia de países como Brasil, la formación de base en la graduación es particularmente relevante, operando como un núcleo fuerte de identidad profesional y académica del graduado y generalmente prolongando este perfil a la formación de posgrado. El abordaje de tipo etnográfico es un punto de atracción significativo de la disciplina, pero considero que al menos en la Argentina la antropología estaba mostrando una sugestiva renovación en el tratamiento de distintos asuntos de tradicional competencia de otras disciplinas entre ellos las violencias de estado en dictaduras y democracia, las reformas de los sistemas de justicia, etc. Y enseñaba un horizonte de trabajo en torno a instalar debates al respecto en el espacio público, u ofrecer estos saberes de investigación para procesos judiciales e institucionales. Estos cruces entre investigación, docencia y compromiso público, entre Derecho y Ciencias Sociales en intervenciones y activismos son un rasgo que el Equipo de Antropología Política y Jurídica de Argentina comparte con el InEAC de Brasil, y que tuve la posibilidad de observar de cerca en mi estancia en dicho instituto.

Las disciplinas que cursé en el Programa de Posgrado durante los meses de mi estadía ("Etnografia e Antropologia do Direito: aspectos metodológicos" dictado por Lucía Eilbaum y “Etnografia em instituições estatais" ofrecido por Ana Paula Mendes de Miranda) y las entrevistas con docentes de la UFF (Marcos Otavio Bezerra) e incluso del PPGAS del Museu Nacional de Rio de Janeiro (con Federico Neiburg y John Comerford) contribuyeron, en una etapa formativa clave para mí, a consolidar mi interés por la disciplina antropológica y encontrar algunas de las herramientas necesarias para un tránsito ya definitivo desde la sociología a la antropología. Era desde ya una gran ventaja el que esos seminarios no fueran de cursado intensivo -es decir dictados en tres o cuatro días seguidos- como la totalidad de los seminarios que había po- 
dido tomar en Argentina, y entonces que semana a semana pudiéramos volver a los temas del programa en una relación reflexiva y de elaboración progresiva de nuestros temas de estudio.

El seminario “Etnografia e Antropologia do Direito: aspectos metodológicos" dictado por Lucía Eilbaum terminó de orientarme en un punto fundamental. Debía confiar en partir, situarme en, girar en torno al "caso" - un caso judicial-, e ir desprendiendo los problemas a partir de allí. Es decir, sin caer en una perspectiva ingenuamente empirista, porque como dice Bourdieu (1995), el objeto de investigación se construye, pude avanzar en un cambio de enfoque metodológico en cuanto a no requerir partir de una descripción exhaustiva del contexto político y del sistema judicial en el que se situaba el proceso a analizar, sino que los asuntos desprendidos del "caso" permitirían decir algo más y de una manera más carnal sobre ese contexto y estructura de relaciones tantas veces analizados por la politología y la sociología política sobre el juarismo santiagueño. La propuesta consistía en fortalecer una perspectiva fundamentalmente etnográfica, que además se apoyara de manera significativa en volver a conversar con parte de la antropología clásica.

Así, la tarea se orientó en esos meses a encontrar dispositivos teóricos ad-hoc para enriquecer el tratamiento de esos problemas construidos/ desprendidos del caso. Durante dicha estadía una de las tareas que ocupó sistemáticamente mi tiempo fue la de una intensa pesquisa bibliográfica, orientada por lxs profesores mencionados arriba y por mi directora, tanto en el Acervo del NUFEP-UFF como en la Biblioteca del Museu Nacional de la Universidad Federal de Rio de Janeiro (hoy tristemente desaparecida por el voraz incendio del Museo a principios de septiembre de 2018) sobre, según registré en un informe: etnografía del derecho y estudios de caso; policía e investigación policial; porosidad entre distintas esferas sociales; antropología de las regulaciones sociales: conflictos, acusaciones, chisme y reputación; etnografías sobre casos de corrupción en Brasil, antropología del don y los intercambios. Cabe apuntar que vivir lejos de Buenos Aires puede dificultar el acceso a bibliografía, y que en 2011, aún con la generalización del acceso a internet, no había tanta disponibilidad como la actual de textos específicos 
-incluso recuerdo no poder conseguir etnografías clásicas-. Por eso, una experiencia internacional en una ciudad grande, también se transformaba en una oportunidad inestimable en ese sentido.

Considero que cierta despreocupación por una elaboración excesivamente teórica previa al trabajo de campo y aún en el texto analítico, es un trazo de los trabajos de investigadores brasileros con los que tuve contacto en esa etapa, aunque también observé una heterogeneidad entre la producción de corte más etnográfico y la elaboración de modelos sociológicos más generales sobre administración de conflictos y matrices comparativas de culturas jurídicas de países como Brasil, Estados Unidos, Argentina, Francia, Portugal...

Esta y posteriores experiencias en Brasil me permitieron conocer más de cerca ciertos debates e incluso irme familiarizando con todo un "lenguaje" que surgió de ese diálogo permanentemente comparativo entre el EAPyJ y el InEAC. Especialmente para quienes trabajamos sobre "provincias" con ciertos rasgos sociológicos que distan de los ideales civilizatorios y modernos ${ }^{9}$ un dilema siempre ha sido el de encontrar cómo y bajo qué condiciones establecer comparaciones y hacer un uso de categorías producidas en referencia a contextos como los de Buenos Aires o Rio de Janeiro. Sabemos que los debates poscoloniales y luego decoloniales han enfatizado en establecer una relación más autónoma con las categorías occidentales (RIVERA CUSICANQUI; BARRAGÁN, 1997) y en que no es posible describir ningún proceso de dominación interna, de gubernamentalidad o ejercicio de poder disciplinar sin comprender que la situación colonial es un patrón estructurador fundamental de las relaciones al interior de nuestros países (ver CASTRO-GÓMEZ, 2000; QUIJANO, 2000). Pero también la antropología, al menos desde la ruptura con el evolucionismo se ha preocupado por construir un saber no etnocéntrico. En este caso creemos que conceptos tales como "modelos de administración de conflictos" de Roberto Kant de Lima (con toda su hibridez entre una influencia del derecho, un enfoque foucoultiano del control social y de los sistemas

\footnotetext{
- La socióloga santiagueña Ana Teresa Martínez se refiere por ejemplo a Santiago del Estero como un caso de "modernización periférica" o de modernización dependiente y nomina a los intelectuales santiagueños como intelectuales "de provincia” (ver, entre otros, Martínez, 2013).
} 
de producción de verdad, y el influjo del sociólogo francés Louís Dumont o la antropología sobre Brasil de Roberto Da Matta); sus variantes como la noción de "campo estatal de la administración de conflictos" de Jacqueline Sinhoretto; el concepto de "sensibilidades legales", tomado de Clifford Geertz y agenciado por varios investigadores brasileros como Daniel Simião; el de "insulto moral" de Luis R. Cardoso de Oliveira, en fin, resultan un ejemplo de esta marca de identidad del instituto brasilero y muestran su versatilidad para hacer permanentemente emerger una empresa comparativa que no descuide las particularidades locales.

Por último, durante esos tres meses participé como parte de mis obligaciones como becaria, de las reuniones semanales coordinadas por Roberto Kant de Lima en el NUFEP, y aún con ciertas dificultades con el idioma vi cómo distintos asuntos operativos, presupuestarios pero también de orden académico eran calurosamente debatidos en esas reuniones. Más que nada quiero referirme a que durante esa residencia no sólo incorporé ciertos conocimientos sino que presencié una modalidad de trabajo intensa y metódica de profesores y personal administrativo en el instituto, con permanentes actividades y proyectos, pero donde también, como me referiré más adelante, había un valor académico - y hasta una obligación- en compartir una cerveza al finalizar una jornada de trabajo.

\section{LA SEGUNDA EXPERIENCIA: CONFORMACIÓN Y CONSOLIDACIÓN DE REDES DE INVESTIGACIÓN}

La segunda etapa de mi vinculación con la academia brasilera se vincula ya con mi trayecto de formación posterior al doctorado ${ }^{10}$. Mi traslado definitivo desde Santiago del Estero a Salta me planteaba la necesidad de definir un tema de estudio que tuviera alguna continuidad con los temas trabajados en Santiago, pero la particularidad del proceso político santiagueño lo hacían de algún modo irreplicable incluso en términos comparativos. Me da-

\footnotetext{
${ }_{10}$ También a través de una beca CONICET de posdoctorado entre 2013 y 2015.
} 
ba cuenta que estaba abandonando al menos provisoriamente los estudios sobre política para colocarme en la otra dimensión de ese trabajo que era su lado más jurídico. Buscaba aproximarme a un objeto que me permitiera volver a algunos de los asuntos que me habían interesado de modo especial en mi recorrido previo. Me atraían los conflictos aparentemente insignificantes entre personas relativamente próximas, que por lo general involucraban asuntos de honor o reputación, intercambios fallidos, chismes y rumores sobre sus causas e identidad de los involucrados, escándalos relativamente públicos y que además se terminaran por canalizar en la justicia. Estaba interesada en la dimensión particularmente antropológica de esas disputas. La Mediación de Conflictos ${ }^{11}$ se presentó como un ámbito propicio para observar esta clase de conflictos "menores", donde acusados y denunciantes estaban envueltos en relaciones de proximidad -eran generalmente vecinos, familiares o conocidos-. Además, por contactos personales y por características propias de este campo que luego analizaría, resultó de relativo fácil acceso. En esta búsqueda me encontré con que la mediación y la conciliación de conflictos ya habían sido dispositivos abordados por investigadores del InEAC desde al menos el año 2003 (particularmente los libros y textos que había podido traer de Brasil en esa primera experiencia formativa fueron antecedentes obligatorios para esos primeros planteamientos). Sin embargo, en Argentina se trataba de un tema completamente inexplorado al menos desde la antropología y la sociología, aún cuando estaba mucho más desarrollada institucionalmente que en Brasil.

En ese estado de situación hacia el año 2012 acertadamente María Victoria Pita, quien me acompañaba de nuevo como directora de beca posdoctoral ${ }^{12}$, convocó a la Dra. Kátia Mello Sento Sé, también investigadora del InEAC y

${ }^{11}$ Una modalidad comprendida entre los llamados Métodos Alternativos de Resolución de Conflictos que propugna por una forma no adversarial de gestión de los conflictos, con la participación voluntaria de las partes y donde un tercero neutral -el mediador- colabora en la construcción de acuerdos consensuados entre las partes.

${ }_{12}$ María Victoria Pita es, como puede apreciarse, un eslabón ineludible en estas articulaciones que describo. Si bien no llegamos a compartir estrictamente este campo de estudio, ha apoyado desde una mirada federal y amplia este trabajo y sigue siendo formadora sobre un modo de pensar, de interrogar los espacios institucionales, barriales y comunitarios en cuanto a las formas de ejercicio y regulación de las violencias y las configuración de estatalidad a partir de estos procesos. 
profesora del Departamento de Política Social e Serviço Social Aplicado de la Universidad Federal de Rio de Janeiro, como mi co-directora. Kátia Mello fue una de las primeras investigadoras de dicho instituto en aproximarse a la mediación judicial (después de los trabajos del grupo coordinado por María Stella de Amorim en los Juizados Esepciais y junto con Jacqueline Sinhoretto) y a lo que ella posteriormente dio en llamar "formas no violentas de administración de conflictos". Simultáneamente a ese incipiente vínculo de trabajo, iniciamos una relación tripartita, que persiste aún hoy, con el Dr. Juan Pablo Matta, profesor y director del Departamento de Antropología de la Universidad Nacional del Centro de la Provincia de Buenos Aires en la ciudad de Olavarría. Matta había trabajado en su tesis doctoral, también financiada por CONICET, sobre conflictos de consumo en una oficina municipal, pero con posterioridad tomó como objeto de investigación los conflictos vecinales tratados en mediación comunitaria en ese mismo centro y asimismo formó el Grupo de Estudios Socioculturales del Conflicto.

Esa relación redundó en múltiples y numerosos productos de investigación, docencia y experiencias de formación e intercambio ininterrumpidos desde el año 2013 hasta la fecha: 1) La proposición y coordinación de grupos de trabajo específicos a la temática en jornadas y congresos científicos internacionales. ${ }^{13}$ 2) Visitas como profesores e investigadores a las distintas universidades de pertenencia ${ }^{14}$. 3) La organización en septiembre de 2018

\footnotetext{
${ }_{13}$ La coordinación de Mello, Matta y mía en 2014 del GT 29 “Estudios etnográficos del conflicto”, en el XI Congreso Argentino de Antropología Social, Rosario (23 al 26 de agosto). En 2015 de Mello, Matta y Carla Villalta del GT: "Sensibilidades jurídicas y sentidos de justicia en la contemporaneidad: un diálogo entre la Antropología y el Derecho" en la XI Reunión de Antropología del Mercosur en Montevideo, Uruguay (del 30 de noviembre al 4 de diciembre). En 2017 de Mello y Matta de la Mesa de trabajo "Políticas Públicas de administração de conflitos: afetos, valores morais, mediações e arranjos sociais" en el XVI Congreso de Antropología en Colombia y el V Congreso de la Asociación Latinoamericana de Antropología, en la Pontificia Universidad Javeriana, Bogotá (6, 7, 8 y 9 de junio). En 2018 la coordinación de Mello y Matta del Open Panel "Public Space, conflict and moral" en el 18th. IUAES World Congress., Florianópolis $(16,17,18,19$ y 20 de julio).

${ }^{14}$ Entre los días 3 y 7 de octubre de 2016 Kátia Mello realizó una misión posdoctoral en el ámbito del ICSOH-CONICET-Universidad Nacional de Salta, dictando la conferencia "Antropología, Políticas Públicas y Demandas de derechos" además de mantener reuniones con tesistas e integrantes del Proyecto de Investigación dirigido por mí. Había hecho lo mismo en la Facultad de Ciencias Sociales de la UNICEN en la ciudad de Olavarría, provincia de Buenos Aires, entre el 19 y 23 de septiembre del mismo año. En el año 2017, entre el 21 de noviembre y el 3 de diciembre Juan Pablo Matta y quien suscribe este artículo participamos de una estancia de investigación con epicentro en la Escola de Serviço Social de
} 
en la UFRJ de un Seminario de investigación con participantes argentinos y brasileros ${ }^{15} 4$ ) la publicación de un dossier y un libro referido a la temática ${ }^{16}$. 5) La integración recíproca en proyectos de investigación con sede en los organismos de ciencia y técnica de origen de cada investigador ${ }^{17}$. 6) Experiencias de trabajo de campo compartido en Olavarría y Salta en septiembre y octubre de 2016. 7) El dictado conjunto de cursos de capacitación en la temática dirigidos a mediadores ${ }^{18}$.

Cabe reflexionar nuevamente sobre la particularidad de que los institutos y universidades asociados en este tejido tengan distinta escala y distinta centralidad en el sistema científico de cada país. El Instituto de Estudos Comparados em Administração Institucional de Conflitos-INCT-InEAC, con sede en la Universidad Federal Fluminense, es un instituto de importante prestigio académico en Brasil; funciona desde 2009 e integra una red nacional e

la Universidade Federal do Rio de Janeiro para trabajo de campo, orientación de alumnos, participación en workshop, reuniones de trabajo y en el V Seminário Internacional do Instituto Nacional de Estudos e Pesquisas em Administração Institucional de Conflitos - INCT-InEAC/NEPEAC/UFF. Estas actividades fueron desenvueltas en el marco del Proyecto de Investigación "Análise comparada de categorias jurídicas em processos sociais de administração de conflitos: democracia, igualdade jurídica, cidadania e demanda por direitos” dirigido por Kátia Mello.

15 Se trató del Seminario "Sociabilidades Urbanas, Espaço Público e Mediação de Conflitos: diálogos com a América Latina”, organizado por el Grupo de Pesquisa sobre Sociabilidades Urbanas, Espaço Público e Mediação de Conflitos - GPSEM - Programa de Pós-Graduação em Serviço Social. Auditório da Escola de Serviço Social/UFRJ (17 e 18 de septiembre).

${ }^{16}$ Se trata del libro Potencialidades e incertezas de formas não violentas de administração de conflitos no Brasil e na Argentina publicado en 2018 bajo la coordinación de Mello, Kátia, S. S., Lupetti Baptista Bárbara Gomes y Filpo, Klever P. L. (Porto Alegre: Evangraf Editora). Y del dossier “Arranjos sociais, mediações e sentidos de justiça” publicado en la Revista de Estudos e Pesquisas sobre as Américas. Centro de Pesquisa e Pós-Graduação sobre as Américas (CEPPAC), Universidade de Brasilia (UnB). Vol. 10, nº 1.

17 Matta y yo integramos desde abril de 2015 el subprojeto "Análise comparada de categorias jurídicas em processos sociais de administração de conflitos: democracia, igualdade jurídica, cidadania e demanda por direitos", dirigido por Kátia Mello en el ámbito del /InEAC/Nufep/UFF. A su vez Kátia Mello y Juan Pablo Matta forman parte como investigadores del proyecto que dirijo en el ámbito del CIUNSa No 2412: "Investigaciones en Antropología Jurídica: Estudios de casos en torno a procesos de producción de derechos, regulaciones sociales y justicia” (Resolución No 306-2016-CCI). Mello y yo integramos el Proyecto "La producción sociocultural del conflicto y sus administraciones" (03/F157), radicado en el Departamento de Antropología Social de la Facultad de Ciencias Sociales de la UNICEN.

${ }_{18}$ Mello, Matta y quien suscribe dictamos el Curso de Capacitación y Actualización "Antropología, Derecho y Mediación. Diálogos y perspectivas”, primero en la ciudad de Azul, Facultad de Derecho, Universidad Nacional del Centro de la Pcia. de Buenos Aires, durante los días 20 y 21 de septiembre de 2016 y luego en Salta, los días 6 y 7 de octubre del mismo año homologado por el Ministerio de DDHH y Justicia del gobierno de la pcia. de Salta, con el aval del ICSOH-CONICET-UNSa y de la Maestría y Especialidad en DDHH de la Facultad de Humanidades. 
internacional de programas de posgrado, grupos de investigación e investigadores de siete estados de Brasil: Rio de Janeiro, São Paulo, Minas Gerais, Pernambuco, Rio Grande do Sul, Bahía y Pará; y de nueve países: África del Sur, Argentina, Canadá, Estados Unidos, Francia, República de Mauricio, México, Portugal e Suiza (http://www.ineac.uff.br). Del lado argentino la Universidad Nacional de Salta (UNSa) y la Universidad del Centro de la Provincia de Buenos Aires (UNICEN) son universidades que se ubican en el contexto de provincias y ciudades de mediana y pequeña escala, nuevamente, distantes de los centros urbanos y administrativos del país ${ }^{19}$.

Hubo, es cierto, políticas de ciencia y técnica positivas que procuraron a mediano plazo cierto crecimiento en cuanto a la cantidad de investigadores, becarios y número de institutos en estas universidades, si bien éstos siguen siendo un número muy inferior al de otros distritos. Cabe aclarar que CONICET no es el único organismo que alberga a investigadores y de hecho lo es en mucha menor medida que el que lo hace el propio sistema universitario. Pero para dar una idea de ese crecimiento y relación entre las provincias en el año 2007, había en Salta 44 Investigadores CONICET, llegando a 117 en

\footnotetext{
19 La provincia de Salta, al noroeste de Argentina, se ubica a $1500 \mathrm{~km}$ de la capital federal, al límite con Bolivia, Paraguay y Chile, y tiene 1.214.441 habitantes según el censo de 2010, estando octava en población respecto todas las provincias y a la ciudad de Buenos Aires. La ciudad de Olavarría se ubica a $370 \mathrm{~km}$ al sudoeste de la capital federal y tiene alrededor de 90.000 habitantes, diseminados en territorio urbano y rural. Sólo se puede llegar hasta allí vía terrestre (no hay por el momento conexión aérea). La Universidad Nacional de Salta, es como en otras provincias del noroeste la única universidad de gestión estatal en la provincia del mismo nombre. Ésta tenía una matrícula de 30.980 alumnos en 2017 y la Facultad de Humanidades 4.717. Mientras que la Facultad de Ciencias Sociales de la UNICEN, con sede en Olavarría, tiene actualmente 300 alumnos activos. La Región Noroeste, hacia el año 2014 ocupaba el quinto lugar entre siete regiones por la cantidad nominal de alumnos universitarios de grado, representando el $9 \%$ de los estudiantes nacionales de carreras de grado. Tiene asimismo el 5,3\% de los estudiantes de posgrado, mientras que la región Metropolitana (que incluye Capital Federal y 24 distritos más) aglomeraba el 48\%. Por su lado, la Universidad Nacional del Centro de la provincia de Buenos Aires, segmenta su oferta educativa entre ciudades como Azul, Tandil, Quequén y Olavarría. En esta última se encuentra la Facultad de Ciencias Sociales, donde está el Departamento de Antropología. Esta fragmentación hace que cada unidad institucional parezca aún más pequeña. Para el caso de la provincia de Buenos Aires, la tasa neta universitaria según el Censo de 2010 era 13,5 y para Salta 10,3, en comparación con la de Capital, de 34,9. Por otro lado la región Bonaerense, donde está la ciudad de Olavarría, es la tercer región argentina (de siete) en cuanto a la cantidad de estudiantes universitarios de grado, representando el $11 \%$ del total nacional (no contamos con el dato específico sobre la zona centro de esa región y especialmente de Olavarría), y tiene además el 11,1\% de los estudiantes de posgrado (Estos datos surgen de un artículo publicado en febrero de 2017 bajo la autoría de Sandra Fachelli y Pedro López-Roldán, con datos de la Secretaría de Políticas Universitarias). La UNSa tenía una matrícula de 30.980 alumnos en 2017 y la Facultad de Humanidades 4717. Mientras que la Facultad de Ciencias Sociales tiene actualmente 300 alumnos activos.
} 
2017, a partir de que este organismo fijara a esta y a otras provincias en el primer orden de prioridad en todas las especialidades y disciplinas para la asignación de becas e ingreso a Carrera de Investigador. En contraste, Capital Federal tiene actualmente 2.936 investigadores CONICET, y toda la provincia de Buenos Aires $2.979^{20}$.

Pero, en fin, lo cierto que seguramente la idea de "periférico" sirvió en algunos casos para aprovechar las oportunidades que otorgaba esa categoría en este contexto expansivo de la ciencia en Argentina. En particular, nuestra experiencia de vinculación transprovincial al interior de Argentina y transnacional con Brasil nos sirvió para afianzar una posición académica a partir de mostrar cierta expertise en el campo de estudios etnográficos sobre mediación, desde la lógica del aporte más general al campo de estudios sobre administración de conflictos. La perspectiva antropológica, con su énfasis en el conocimiento local (GEERTZ, 1994), nos lleva a re-pensar los límites entre centro y periferia (DAS; POOLE, 2008, p. 20), pues entendemos que las manifestaciones de las burocracias, el derecho y los modos en que se tejen y se gestionan los conflictos son tan singulares -y tan otros para esta clase de comprensión- en Rio de Janeiro, Olavarría y Salta. Cabe pensar cómo esta postura disciplinar se fortalece con una postura de cierto modo política.

Esta relación entre personas pero que a su vez movilizaba instituciones más amplias de distinto porte, se dio de un modo particularmente horizontal, enlazando también distintas generaciones: una investigadora en un nivel más avanzado de su trayectoria y dos “jóvenes" de entre 30 y 40 años recién graduados de su doctorado, con lugares más o menos consolidados en sus universidades. De este modo cada una de las partes gestionó espacios institucionales propios para activar estas alianzas y participó prácticamente de igual a igual en aquellos propuestos por sus interlocutores. Llamamos la atención sobre esto, en el trasfondo de sociedades ciertamente jerarquizadas, segmentadas y desiguales pero que también tienen tradiciones de impugnación de esas jerarquías ${ }^{21}$.

${ }_{20}^{20}$ Fuente: Base de datos de CONICET, Gerencia de Recursos Humanos, Oficina de Información Estratégica de RRHH (CONICET, 2017).

${ }^{21}$ Siempre es útil volver a la contraposición entre el rito autoritario Você sabe com quem está falando? con el que Roberto Da Matta sintetiza las relaciones jerárquicas y personales que caracterizarían la sociedad 
En esta etapa de producción, logré movilizar quizá más conscientemente varios de los conceptos y del "lenguaje" con los que había tenido contacto en la etapa previa, y que además, se acomodaban más íntegramente a la nueva problematización. Al contrario de la investigación anterior donde un proceso histórico y social muy singular parecía irradiar con facilidad, en la indagación sobre el dispositivo de la mediación la particularidad del proceso salteño y argentino a veces quedaba opaco frente al modelo teórico producido para el contexto brasilero. En parte creo que esa fue una debilidad que tienen algunos de mis primeros trabajos sobre el tema. Ciertamente influida por esos antecedentes mi trabajo se concentró menos en la cuestión del conflicto que en el proceso institucional de la mediación en nuestra región. Aspectos como el proceso de constitución de un campo específico dentro del campo estatal de la administración de conflictos en Argentina (MATTA; GODOY, 2016); ciertas disputas institucionales y de categorías en el desarrollo de la mediación penal en Salta (GODOY, 2016); la afirmación de un tipo de "profesión", la del mediador, dentro del campo de la justicia (GODOY; MATTA, 2018), son ejemplos de los temas abordados.

Michel Callon (1995) en lo que llama una "sociología de la traducción" entiende que simultáneamente a que se produce el proceso de problematización o de producción de conocimiento científico se construye una red de relaciones entre distintos actores, que en nuestro caso incluye a los mediadores y otros agentes de ese espacio social de análisis, probablemente a sus "expedientes" y a los usuarios del sistema, pero también supone alianzas y enrolamientos dentro de la comunidad científica. Es decir que la comunidad científica no existe necesariamente de antemano al conocimiento científico y tampoco esos sujetos de estudio que, a través de ese proceso de conocimiento pueden emerger como verdaderos grupos ${ }^{22}$.

brasilera, o más bien carioca, y el rito impugnador de las jerarquías Y mi qué me importa, que esboza Guillermo O 'Donell para hablar de una sociedad argentina -acaso porteña- pretendidamente más igualitaria. Como esta alianza académica comprendió regiones muy diferentes de Argentina estos modelos tienden a complejizarse, hacerse más híbridos y relativos.

${ }^{22}$ Lo específico de esta propuesta además es que las alianzas que se producen en el proceso de investigación o tecnológico incluyen no sólo a entidades sociales sino también naturales: en su artículo muestra lo 
Pero ¿de qué están hechos esos intercambios y qué los motivan para que se mantengan en el tiempo? Ya Weber, en "La ciencia como profesión" se preguntaba por el sentido o el valor de la ciencia para quien se dedica a ella. $\mathrm{Y}$ encontraba que su sentido si bien se vinculaba a lo técnico y a la validez de las reglas de la lógica, tenía un sustrato adicional. La actividad científica como profesión -que según este clásico no era distinta a la del artista por ejemplo- requiere de una vocación o disposición interior (WEBER, (2001[1919]), p. 60); una vivencia de la ciencia, pasión, inspiración, alma, que distingue a la personalidad del científico del mero especialista. Una creencia (no usa exactamente este término) en que su resultado es importante, en el sentido de que "merece la pena conocerlo" (p. 73). Esta creencia en la necesidad del conocimiento que producimos y esta pasión por lo que hacemos en el mismo proceso en el que lo hacemos motiva a cada integrante de esta red de intercambios.

Ahora bien, Bourdieu por su parte entiende que la práctica científica está animada por un cálculo interesado -un tanto inconsciente según este modelo teórico- en cuanto a las posibilidades de acumulación de capital científico, es decir, de reconocimiento, si bien esta búsqueda es siempre en la práctica fuertemente negada en nombre del ideal del desinterés (Bourdieu, 2003, p. 97). En las alianzas académicas por supuesto hay algo o mucho de esto, como en el intercambio de dones, donde todo acto generoso esconde "la ambición de asegurarse un poder, un dominio duradero sobre el beneficiario" (p. 98). Pero también lo vivimos, y esto es parte de la eficacia de nuestra práctica, como un conjunto de actos generosos y voluntarios, como que tenemos la voluntad de estar juntos. En este sentido volviendo a Weber que reconocía la acción social en su dimensión de valor, y en la afinidad valorativa que produce relaciones sociales y hasta regímenes de valor completos -como el capitalismo- también introducía en ella el ideal afectivo (WEBER, 2014[1922]). El reciente libro publicado en Argentina por Ana Abramowski y Santiago Canevaro llama a pensar los afectos en distintos universos sociales -el del trabajo, el de las religiosidades, el educativo, el de la militancia política, la maternidad...- como

complejo del proceso social de "interesamiento", verdaderas batallas de seducción y acorralamiento de colegas científicos, de pescadores y también de las vieiras que los científicos proponen reproducir. 
manera de explorar las "zonas desprolijas y contradictorias en las que se gestan lazos e identidades, se construyen sensibilidades y se generan sociabilidades" (ABRAMOWSKI; CANEVARO, 2017, p. 15).

La investigación no está ajena a este componente. Lo destaca el precioso texto de Roberto Da Matta sobre el anthropological blues, es decir, la experiencia donde en la situación o el descubrimiento etnográfico las emociones y los sentimientos, la subjetividad y la carga afectiva, los dolores, los amores, las pérdidas, las ansiedades y los miedos se cuelan en el proceso intelectual. ¿Por qué no hacer alcanzar estos componentes a toda la situación académica?: "me veo frente a gente de carne y hueso. Gente buena y antipática, gente sabia y estúpida, gente fea y bonita" (DA MATTA, 2017, p. 228) cuando estoy frente a las personas que son mis directores, orientadores pares y colegas, frente al contacto humano que hace al oficio. Es decir, hay afinidad intelectual, empatía y también afectiva en estos vínculos "internacionales", a veces incluso más intensas que con las personas de nuestros propios lugares de trabajo, y que cultivada con el tiempo facilitan su permanencia. Esta manifestación afectiva también nos recuerda que nuestros encuentros académicos transprovinciales o transnacionales son viajes físicos y de alteridad. $Y$ ahí a los argentinos nos impresiona y deleita esa "forma carioca" propiamente académica donde el trabajo intenso no descuida las celebraciones recreativas, la cerveza, el vino y la comida, las expresiones de amistad que prolongan el aula al bar.

En definitiva, estos vínculos que describimos atravesaron ese contexto benéfico para la ciencia y técnica en nuestra región, y luego la incertidumbre de una transición hacia un proyecto de raigambre neoliberal con contracción estatal y de las políticas en esta materia. Hubo iniciativas de nuestra red a partir de 2016 que fueron sostenidos sin recurso alguno -como la experiencia posdoctoral de Kátia Mello en Olavarría y en Salta ese año-, y otras que con recursos residuales adquirieron un nuevo significado en ese escenario. El Seminario "Sociabilidades Urbanas, Espaço Público e Mediação de Conflitos: diálogos com a América Latina", celebrado en el Auditório da Escola de Serviço Social de la UFRJ en septiembre de 2018 y organizado por Kátia Mello y Patricia Farías, significó para estas organizadoras una apuesta de resistencia 
a las propuestas de desgaste y deslegitimación de las universidades públicas, "una manera de sentirse "fuertes" frente a esos embates y encontrarnos afectiva y colectivamente". Dicho seminario se dedicó a la memoria de Marielle Franco, socióloga, militante negra y feminista asesinada en Rio en marzo de ese año, y del Museu Nacional de la UFRJ, incendiado casi por completo también debido en parte al deterioro de la inversión en políticas culturales y educativas en dicho país.

\section{PALABRAS DE CIERRE}

El presente ensayo intentó reconstruir la trayectoria académica de una investigadora que viene de universidades del noroeste argentino, en aquellos aspectos que guardan relación con experiencias de articulación con la academia brasilera en el campo de la antropología jurídica y de la administración de conflictos. En esta exposición se aprecia una combinación compleja entre iniciativas personales y grupales y condiciones más amplias que hicieron posibles esas experiencias de vinculación. A la vez, nos preguntamos por el modo en que las biografías sociales y académicas son afectadas por esas coyunturas.

De ningún modo quisiera que lo relatado aquí sea leído como una trayectoria personal lineal y exitosa. A pesar de algunos logros importantes, la mayor parte del tiempo me acompañaron dificultades en cuanto a la continuidad de fuentes laborales ligadas a la investigación y a la docencia universitaria y estuve incluso fuera del sistema CONICET en Argentina. Más allá de traspiés personales, esto habla también de sistemas públicos que no han logrado salir de la fragilidad y la precariedad.

Las alianzas académicas con personas de otras provincias y de Brasil, que como dije, en varios casos se transformaron en vínculos perdurables de amistad, me obligaron en esos momentos de vulnerabilidad, a mantenerme proactiva y a disminuir la carga de atribución personal de las dificultades. Es decir, estas alianzas tienen una función fundamental que tal vez no he subrayado lo suficiente: permiten contrarrestar corrientes de desánimo - personales y co- 
lectivas-, tomar suficiente distancia de ellas o ponerlas en su sitio en el consorte de procesos sociales más amplios.

Pero cuando la inversión nacional en desarrollo científico de un país se resiente, seguramente hay otras áreas del tejido social que están siendo también deterioradas. Por eso nuestra preocupación en el último tiempo ha sido la de observar en nuestros países cierto recrudecimiento de las formas punitivas de control social, en desmedro de alternativas que no requieren del uso de la fuerza. En el último libro que resulta de este sistema de colaboración entre investigadores argentinos y brasileros se reflexionaba:

"las sociedades latinoamericanas están pasando por procesos de desintegración de las políticas públicas democráticas de control del orden social y de administración de conflictos en diferentes ámbitos de la vida social. Cada vez es más necesario discutir y proponer políticas públicas que consideren estrategias diferentes al uso de la fuerza y de la violencia ¿es posible administrar conflictos sin recurrir al uso de la fuerza bruta? Es con esta preocupación que este libro pretende comprender cómo se articulan diferenciadas formas de administración de conflictos en Brasil y Argentina"23.

\section{REFERENCIAS}

1. ABRAMOWSKI, Ana; CANEVARO, Santiago (comp.). Pensar los afectos: aproximaciones desde las ciencias sociales y las humanidades. Buenos Aires: UNGS, 2017.

2. BOURDIEU, Pierre. La práctica de la antropología reflexiva. In: BOURDIEU, Pierre. Respuestas: por una antropología reflexiva. México, DF: Grijalbo, 1995. p. 159-196.

3. BOURDIEU, Pierre. Un mundo aparte. In: BOURDIEU, Pierre. El oficio de científico: ciencia de la ciencia y reflexividad. Barcelona: Anagrama, 2003. p. 63-148.

${ }^{23}$ MELLO; LUPETTI BAPTISTA; FILPO, 2018. Contratapa. Traducción propia, en portugués en el original. 
4. CALLON, Michel. Algunos elementos para una sociología de la traducción: la domesticación de las vieiras y los pescadores de la Bahía de Brieuc. In: IRANZO, Juan Manuel; BLANCO, J. Ruben., GONZÁLEZ DE LA FE, Teresa; TORRES, Cristobal; COTILLO, Alberto (comp.). Sociología de la ciencia y la tecnología. Madrid: CSIC. 1995. p. 259-282.

5. CASTRO GÓMEZ, Santiago. Ciencias sociales, violencia epistémica y el problema de la "invención del otro". In: LANDER, Edgardo (comp.) La colonialidad del saber: eurocentrismo y ciencias sociales: perspectivas latinoamericanas. Buenos Aires: Clacso, 2000. p. 145-161.

6. CONICET. Indicadores destacados. Buenos Aires, 2018. Disponível em: https://bit.ly/2MReWi6. Acesso em: 19 ago. 2018.

7. CONICET. Investigadores: investigadores por área de conocimiento 2017. Buenos Aires, 2017. Disponível em: https://bit.ly/2DdhimX. Acesso em: 19 nov. 2018.

8. DA MATTA, Roberto. El oficio del etnólogo o cómo tener "Anthropological blues". In: BOIVIN, Mauricio; ROSATO, Ana; ARRIBAS, Victoria. Constructores de otredad: una introducción a la antropología social y cultural. Buenos Aires: Eudeba, 2007. p. 227-236.

9. DAS, Veena; POOLE, Deborah. El estado y sus márgenes: etnografías comparadas. Cuadernos de Antropología Social, Buenos Aires, n. 27, p. 19-52, 2008.

10. FACHELLI, Sandra; LÓPEZ-ROLDÁN, Pedro. Análisis del sistema universitario argentino: una propuesta inicial de indicadores. Barcelona: Universitat Autònoma de Barcelona, 2017. Disponível em: http://ddd.uab.cat/record/171528. Acesso em: 5 fev. 2019.

11. GEERTZ, Clifford. Conocimiento local: hecho y ley en la perspectiva comparada. In: GEERTZ, Clifford. Conocimiento local: ensayos sobre la interpretación de las culturas. Barcelona: Paidós, 1994. p. 195-287.

12. GODOY, Mariana Inés. Los regalos de Margarita: conflictos, trama política y justicia en el juarismo santiagueño. Santiago del Estero: EDUNSE, 2014.

13. GODOY, Mariana Inés. Delito, conflicto: sensibilidades legales y trama institucional en el campo de la mediación penal en Salta-Argentina. Revista del Museo de Antropología, Córdoba, v. 9, n.1, p. 57-68, 2016.

14. GODOY, Mariana Inés; MATTA, Juan Pablo. Creencia y eficacia en la profesión del Mediador de conflictos: una aproximación desde la teoría antropológica de la magia. In: MELLO, Kátia S. S.; LUPETTI BAPTISTA, B. G.; FILPO, Klever P. L. (org.). Potencialidades e incertezas de formas não violentas de administração de conflitos no Brasil e na Argentina. Porto Alegre: Evangraf, 2018.

15. MARTÍNEZ, Ana Teresa. Cultura, sociedad y poder en la Argentina: la modernización periférica en Santiago del Estero. Santiago del Estero: EDUNSE, 2013. 
16. MATTA, Juan Pablo; GODOY, Mariana Inés. El movimiento de Mediación en Argentina: procesos, tensiones y afirmaciones. Revista de Estudos e Pesquisas sobre as Américas, Brasília, DF, v. 10, n. 1, p. 1-23, 2016.

17. MELlO, Kátia S.S.; LUPETTI BATISTA, B. G.; FILPO, Klever P. L. (org.). Potencialidades e incertezas de formas não violentas de administração de conflitos no Brasil e na Argentina. Porto Alegre: Evangraf, 2018.

18. QUIJANO, Aníbal. Colonialidad del poder, eurocentrismo y América Latina. In: Lander, Edgardo (comp.). La colonialidad del saber: eurocentrismo y ciencias sociales: perspectivas latinoamericanas. Buenos Aires: Clacso, 2000. p. 201-246.

19. RIVERA CUSICANQUI, Silvia; BARRAGÁN, Rossana (comp.). Debates poscoloniales: una introducción a los estudios de la subalternidad. La Paz: Historias, 1997.

20. WEBER, Max. La ciencia como profesión. 2. ed. Madrid: Espasa Calpe. 2001[1919].

21. WEBER, Max. Economía y Sociedad. México, DF: Fondo de Cultura Económica, 2014[1922]. 